\title{
A Hybrid Optimization for Improving Network Performance in Scalable Mobile Ad Hoc Network
}

\author{
Rangaraj Jayavenkatesan ${ }^{1 *}$ and Anitha Mariappan ${ }^{2}$ \\ 'Department of Electronics and Communication Engineering, Annamalai University, \\ Chidambaram - 608002, Tamil Nadu, India; jsrd_jsrd@yahoo.co.in \\ ${ }^{2}$ Department of Electrical Engineering, Annamalai University, \\ Chidambaram - 608002, Tamil Nadu, India; vishanitha@yahoo.com
}

\begin{abstract}
Objectives: To optimize the throughput of the network for ensure the seamless connection in a decentralized mobile ad hoc network. Methods/Statistical Analysis: This paper proposes a hybrid optimization technique (ACO-FDR PSO) of Ant Colony Optimization (ACO) integrated with Fitness Distance Ratio Particle Swarm Optimization (FDR PSO) to optimize throughput. ACO algorithm identifies unique path in the network based on higher pheromone concentration. FDR PSO optimizes throughput based on Packet Delivery Factor (PDF). Since FDR PSO employs N-best particle in the velocity update equation, the drawback of PSO such as premature convergence and getting stuck into local optimum can be prevented. Findings: Simulations performed with network simulator 2 on a 100 node network and the metrics, throughput, delay, overhead, energy and packet delivery ratio have been analyzed. Through the results it has been showed that the proposed hybrid technique achieves better results than standard AODV, ACO, PSO and ACO-PSO techniques. Application/ Improvements: The proposed technique facilitates reliable transmission in emergency situations like military application, disaster management etc, where data intensive wireless networks are required.
\end{abstract}

Keywords: Ant Colony Optimization (ACO), Fitness Distance Ratio Particle Swarm Optimization (FDR PSO), Mobile Ad-Hoc Networks (MANET), Optimization, Particle Swarm Optimization (PSO), Position and Velocity Update

\section{Introduction}

Mobile Ad-Hoc Networks (MANET) is seamless constituent of a wireless network having self contained nodes in it. The mobile nodes are distributed such that the nodes within the range can form a network among them and can interface with each other directly within their range. If the mobile node happens to be out of range, then the node communicates via intermediate nodes 1 . The mobile networks change dynamically since the nodes move in different directions, different speeds, and this network topology changes unpredictably ${ }^{2}$.

To predict the mobility of the nodes, the mobility models come into existence. The MANET mobility models illustrate the activity model of mobile nodes. The mobility model depicts the consequential task in establishing the recital of the models and to follow the movement of mobile nodes in an intelligent route. The mobile node model includes the prediction of location and the acceleration of the node since it varies frequently. The advantage of mobility models includes the solutions to routing overhead and issues related to the update of routing table, dynamic topology and the issues related to repeat path disconnections arising from the mobility induced route changes ${ }^{3}$.

Sharma et. al., studied diverse mobility models and their characteristics for independent nodes. Reactive protocol provides better performances in terms of Packet Delivery Ratio (PDR), delay and routing load than other conventional protocols.

A novel approach for GPS utilizing ACO which provides reliable communication with lower delay, route cost and higher throughput when compared to other routing protocols $s^{4}$.

${ }^{*}$ Author for correspondence 
In $^{\frac{5}{5}}$ authors studied the dynamic topology change of networks consisting mobile nodes, varying the speed and position of mobile nodes and node distributions. They achieved better mobility despite variable speed due to increased node density.

$\mathrm{In}^{6}$ authors employed ACO to define a routing protocol that supports fault tolerance. It deploys both forward and backward ants to decide a path with high preference based on its availability, bandwidth and delay metrics. This method excludes fault nodes during evaluation which retains QoS.

$\mathrm{In}^{\mathrm{Z}}$ authors proposed component based framework containing joint routing, channel assignment and flow aware algorithms for route optimization. It increases the routing throughput and improves the route selection by observing the congestion of the network and discarding routing links in and around the regions.

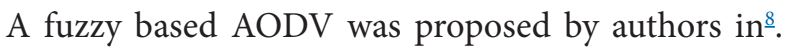
Consider residual energy of all nodes and their mobility pattern besides distance in determining the travelling path from source to the destination. Fuzzy AODV selects trusted nodes to retain the stability of the path and trusted nodes are preferences if they posses higher residual energy and lesser speed.

A hybrid technique (GA-PSO) based on Genetic Algorithm (GA) and Particle Swarm Optimization (PSO) was proposed by authors in ${ }^{2}$ for multicast routing that selects the best outcomes of hybrid algorithm to improve multicast routing on comparison with other multicast routing protocols, GA-PSO improves packet delivery ratio, jitter and decreases delay.

$\operatorname{In}^{10}$ authors studied various ant colony based adaptive routing algorithms for high scalability, adaptability, multicast support, minimizing routing delay and overhead, improving Qos. The algorithms are dependent upon positive and negative feedback observed by the ants in the routing path.

In $\stackrel{11}{ }$ authors studied the working of Ad-Hoc on Demand Distance Vector (AODV) protocol and Destination Sequence Distance Vector (DSDV) protocol. Compared to DSDV, AODV minimizes end-to-end delay, evades loops in routing path, support to mobility providing higher packet delivery ratio and less power consumption.

$\mathrm{In}^{12}$ authors proposed a Random Early Detection (RED) which incorporates AODV to detect and control congestion. RED speculates the queue length and its average utilization for early detection of congestion. AODV alerts the source to curtail the data transmission and thereby avoiding the escalation of packet drop. RED with AODV improves PDR by minimizing packet loss.

$\mathrm{In}^{\underline{13}}$ authors proposed Quality of services enabled Ant colony based Multipath Routing (QAMR) algorithm which is highly adaptive and scalable. QAMR can reduce delay, bandwidth and hop count by selecting the path by its path reference probability.

In ${ }^{14}$ authors employed a hybrid technique based on ACO and PSO. ACO used to find unique path flanked by source and destination, PSO minimizes delay, cost and power consumption.

Though many authors employed PSO technique for routing problem, it is prone to premature convergence and getting struck into local optimum. Hence to evade these tribulations a hybrid optimization technique (ACO-FDR PSO) is proposed for MANET routing problem, ACO selects unique path in the network and FDR PSO maximizes the throughput. Unlike PSO, in FDR PSO, average and best fitness differ for many more iterations which prevent the premature convergence and getting struck keen on local optima problem ${ }^{15}$.

This paper highlights hybrid optimization technique (ACO-FDR PSO) along with traditional routing protocol (AODV) under pursue mobility model. The optimization technique aims at improving the throughput of the network. The results obtained are compared with conventional routing protocol and other optimization techniques with respect to the selected parameter.

\section{Problem Formulation}

Decentralized networks gain access to Quality of Services (QoS) through control and data messages. Routing protocols are vital in minimizing the bridge between increasing routing demand and network optimization. Conventional routing protocol do not have enough flexibility to satisfy new routing demand which force network administrator to augments throughput in which satisfies the needs of those user. As the protocols adapt to a fewer optimization metrics, performance enhancement is considered as a tradeoff in these networks. To improve the purpose of routing and protocol features, various optimization techniques have been integrated with the routing protocols. The integrated approaches lag in resolving pre-convergence issues or require multiple iterations in attaining a stable optimization. The proposed hybrid (ACO-FDR PSO) technique improves the network performance by determining optimal path and improve throughput. 
Throughput function $\left(T_{\mathrm{p}}\right)$ is represented as:

$$
\mathrm{T}_{\mathrm{P}}=\operatorname{Max} \sum_{\mathrm{i}=1}^{\mathrm{n}} \frac{\mathrm{P}_{\mathrm{n}}(\mathrm{i})}{\mathrm{t}(\mathrm{i})}
$$

Where $\mathrm{P}_{\mathrm{n}(\mathrm{i})}$ is the numbers of packets successfully sent and $t(i)$ is the total time at which transmission takes place.

\section{ACO Hybrid FDRPSO}

ACO hybrid FDR PSO assimilates ACO into FDR PSO; the process is divided into optimal path selection and best solution path. Optimal path selection is preceded using ACO whose inputs are fed into FDR PSO. FDR PSO checks for the best path based on throughput function evaluated among the ACO generated paths with their corresponding best position and velocity.

\subsection{ACO}

ACO has the capacity to locate the optimal path, by considering the ants as an agent which is forwarded randomly from one node to another. While walking, the ants put some sum of pheromone on the link of the path. Next generation of ant are attracted by the high pheromone concentration to achieve the optimal path. By adjusting the update mechanism, the pheromone values are dynamically adjusted $\frac{16,17}{6}$. The probability of an ant ' $\mathrm{k}$ ' choosing node ' $j$ ' from ' $i$ ' at an time's' can be written as,

$$
P_{i j}^{k}(t)=\frac{\left[\tau_{i j}(t)\right]^{\alpha} \cdot\left[\eta_{i j}\right]^{\beta}}{\sum_{l \in N_{i}^{k}}\left[\tau_{i l}(t)\right]^{\alpha} \cdot\left[\eta_{i l}\right]^{\beta}} \text { if } j \in N_{i}^{k}
$$

Where,
Prior available heuristic value, $\eta_{i j}=\frac{1}{d_{i j}} . \alpha$ and $\beta$ are two parameters which determines the relative influence of the pheromone trace and heuristic objective function. Possible neighborhood of ant $k, N_{i}^{k}$.

Hop count completed ants updates the pheromone value. The pheromone update of the ants is written as

$$
\tau_{\mathrm{ij}} \leftarrow(1-\rho) \cdot \tau_{i j}+\sum_{n=1}^{m} \Delta \tau_{i j}^{n}
$$

Where

$\rho$ - Evaporation rate, $m$ - number of ants and $\Delta \tau_{i j}{ }^{n}$ pheromone quantity laid on link $(\mathrm{i}, \mathrm{j})$ by the $\mathrm{n}^{\text {th }}$ ant.

$\Delta \tau_{i, j}{ }^{n}=\frac{1}{H_{n}}$ if ant $\mathrm{n}$ travels on link $\mathrm{i}, \mathrm{j}$

Where $H_{n}$ is the hop count of the $\mathrm{n}^{\text {th }}$ ant

Once every ant has finished their tour for one time, there will be multiple paths from source to destination. In all paths, pheromone values are calculated and the path with higher pheromone value is considered as optimal path which is used for further communication.

\subsection{FDR PSO}

FDR PSO optimizes throughput in the optimal path by selecting maximum PDF of all the nodes in the network. The process applied for multipath to enhance throughput. Successive higher PDF nodes are selected for transmission. The PDF of a node represented as:

$$
\mathrm{PDF}=\left(\frac{\mathrm{D}_{\mathrm{R}}}{\mathrm{D}_{\mathrm{S}}}\right)
$$

Where $D_{R}$ is the data received and $D_{S}$ is the data sent.

Premature convergence problem of PSO is prevented in FDR PSO by considering $n$ best particle which maximizes throughput. Fitness distance ratio of throughput is indicated as

$$
\frac{T_{p}\left(P_{i}\right)-T_{p}\left(X_{i}\right)}{\left|P_{i d}-X_{i d}\right|}
$$

$T_{p}\left(P_{i}\right)$ is the throughput of prior best position of the particle, $T_{p}\left(X_{i}\right)$ is the throughput of current particle position, $P_{i d}$ is the prior best position of the particle, and $X_{i d}$ is the current particle position.

Hence the velocity update equation of the particle presented as below:

$V_{i d}^{K+1}=\left(w^{*} V_{i d}^{k}\right)+a_{1} r_{1}\left(P_{i d}-X_{i d}\right)+a_{2} r_{2}\left(P_{\text {gid }}-X_{i d}\right)+a_{3} r_{3}\left(P_{n d}-X_{i d}\right)$

Position update equation of the particle is computed using:

$$
\mathrm{X}_{i d}^{\mathrm{k}+1}=\mathrm{X}_{\mathrm{id}}^{\mathrm{k}}+\mathrm{V}_{\mathrm{id}}^{\mathrm{k}+1}
$$


Where, $\mathrm{P}_{\text {id }}$ is the best previous position (pbest) of the particle, $\mathrm{P}_{\text {gid }}$ is the global best position (gbest) of the particle, $\mathrm{X}_{\text {id }}$ and $\mathrm{V}_{\text {id }}$ are the current values of position and velocity of the particle, $a_{1}, a_{2}, a_{3}$ are the acceleration co-efficient, $r_{1}$, $r_{2}, r_{3}$ be the random numbers between zero to one, $\mathrm{W}$ is inertia weight $\frac{18}{}$.

\subsection{Algorithm of ACO - FDR PSO}

The algorithmic steps for the proposed MANET routing based on ACO-FDR PSO is explained as follows:

Step 1: Initialize the ants in the network,

Step 2: The ants visit each of the nodes to reach the destination and update their pheromone value using equation (3),

Step 3: Select an optimal path to transmit data to the destination,

Step 4: Compute FDR throughput using equation (1) and update Nbest,

Step 5: Compute position and velocity of the current FDR agent using equation (7) and (8),

Step 6: If all the updates are performed then end the process, and

Step 7: If no optimal solution is achieved after step 2 then move to next agent and repeat from step 3 to 6 to perform optimization.

\section{Simulation Background}

We consider 100 nodes that are randomly placed in a network scenario. Nodes in the network follow pursue mobility model. The nodes are linked with one or more neighbors in order to communicate. The transmission range is fixed for each node. Path establishment between nodes is enabled by AODV. The paramerters used for the analysis of proposed rounting is shown in Table 1.
Table 1. Simulation parameters and their values

\begin{tabular}{ll}
\hline Simulation parameters & Assigned value \\
\hline Number of nodes & 100 \\
Network area & $1000 \mathrm{~m} \times 1000 \mathrm{~m}$ \\
Transmission range & $550 \mathrm{~m}$ \\
Data rate & $512 \mathrm{~Kb}$ \\
Simulation time & $100 \mathrm{~s}$ \\
Number of packets & 1500 \\
Mobility model & Pursue \\
\hline
\end{tabular}

\subsection{Results and Discussion}

The proposed hybrid technique is employed on 100 node network to illustrate its effectiveness in maximizing throughput in the optimal path selected. The performance of proposed hybrid (ACO - FDR PSO) technique is compared against AODV, ACO, and ACO-PSO. The proposed technique is evaluated using the network parameters throughput, packet delivery ratio, end-to-end delay, utilized energy and overhead. The proposed technique is tested using NS-2 simulator ${ }^{19}$ and the obtained results are given in Table 2.

\subsection{Impact on Throughput}

Figure 1 shows the variation of throughput obtained by various techniques. The results show that proposed ACO-FDR PSO technique has better performance when compared with conventional protocol AODV, ACO, ACO-PSO techniques. Specifically, throughput in ACOFDR PSO is 2.52 times better than AODV, 1.95 times better than ACO, and 1.01 times better than ACO-PSO.

Table 2. Comparison of different techniques with respect to various parameters

\begin{tabular}{lllll}
\hline PARAMETERS & AODV & ACO & ACO-PSO & ACO-FDRPSO \\
\hline Throughput (Kbps) & 114 & 186 & 356.1 & 363.2 \\
Delay (ms) & 108.475 & 37.589 & 38.682 & 40.907 \\
PDR (\%) & 89.98 & 62.08 & 79.81 & 90.98 \\
Overhead (\%) & 16.08 & 14.41 & 15.04 & 20.04 \\
Energy (J) & 16.68 & 18.87 & 17.2 & 19.34 \\
\hline
\end{tabular}

Table 1, it is evident that throughput is maximized by the proposed technique and PDR is also high when compared with other techniques. 
The proposed technique maximizes throughput as it selects best path based on higher PDF generating nodes.

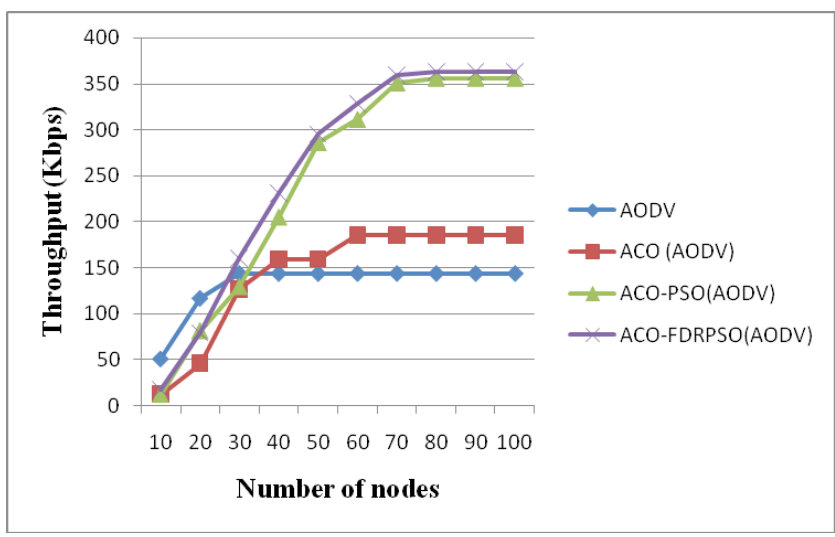

Figure 1. Throughput comparison of conventional routing protocol with AI techniques.

\subsection{Impact on Delay}

Figure 2 shows delay of ACO-FDR PSO in comparison to other techniques. ACO-FDR PSO reduces the delay than other techniques by adopting proper route setup and maintaining it. Route maintenance play a pivotal role in reducing the delay. If route setup is not satisfactorily maintained and if there is a broken path, then the intermediate node may inform it to the sender node which increases the delay. But in the proposed technique, route setup is properly maintained which reduces the delay very much.

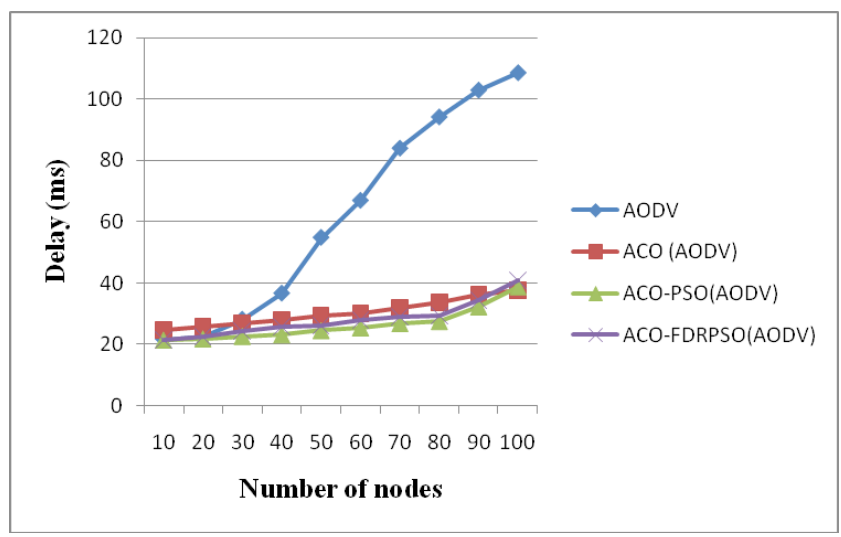

Figure 2. Delay comparison of conventional routing protocol with AI techniques.

\subsection{Impact on Packet delivery ratio}

Figure 3 illustrates PDR of the proposed ACO-FDR PSO with AODV routing protocol. PDR is directly pro- potional to the throughput of the network. It is clearly observed that our proposed technique has significantly better packet delivery ratio than that of other techniques, especially packet delivery ratio is 1.01 times higher than that of conventional AODV protocol.

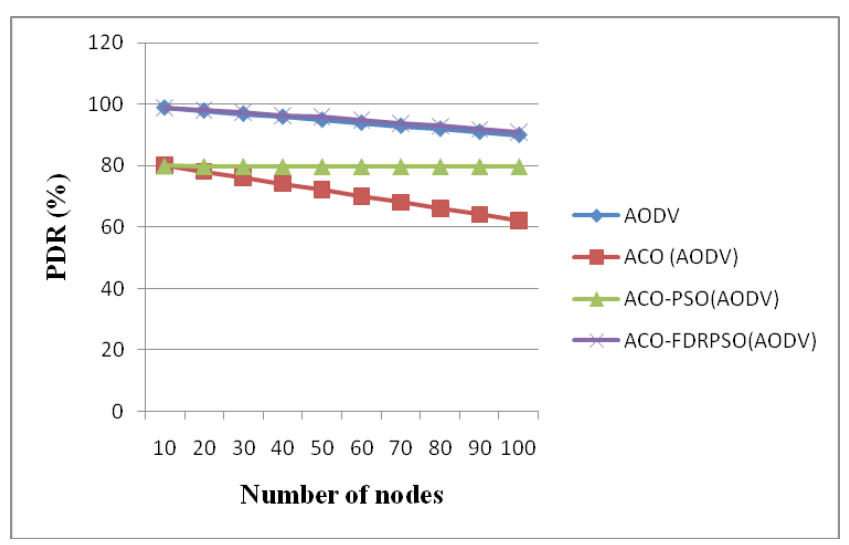

Figure 3. Packet delivery ratio comparison of conventional routing protocol with AI techniques.

\subsection{Impact on overhead}

Figure 4 shows the comparison between various techniques for overhead. Link failures are common when node mobility is high, resulting in frequent routing which increases the overhead in the network. During the route setup, more amounts of control packets are vital for searching and monitoring new paths. Because of the regular update of the path, ACO-FDR PSO technique requires more control packets and hence overhead is better than that of AODV, ACO and ACO-PSO techniques.

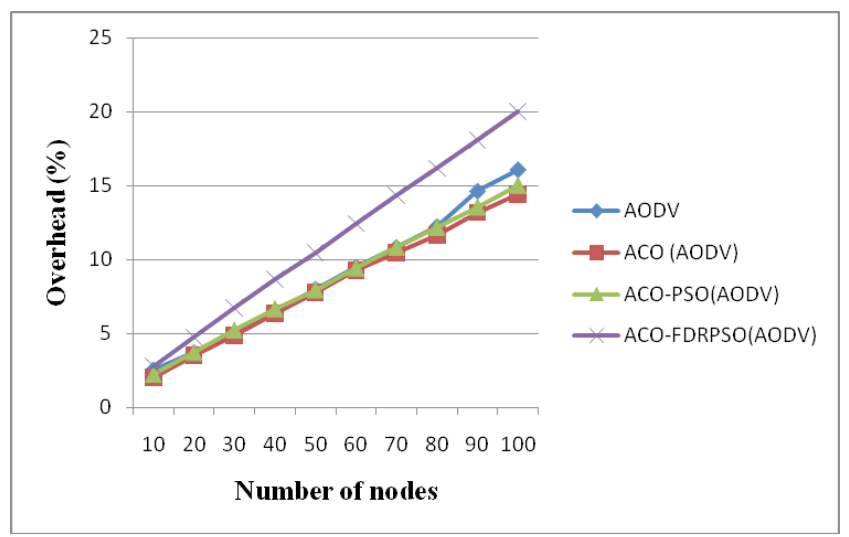

Figure 4. Overhead comparison of conventional routing protocol with AI techniques. 


\subsection{Impact on Energy}

Figure 5 shows energy utilization of conventional protocol and that of proposed protocol. As the node data handling capacity increases, energy consumed also increases. Due to frequent change of node caused by varying mobility patterns, energy consumption increases. In AODV, the number of routing and packet transfer count are less, it utilizes only lesser amount of energy. But in case of hybrid optimization techniques, the energy utilization varies due to higher PDF node selection process which hikes the energy utilization from the conventional protocol.

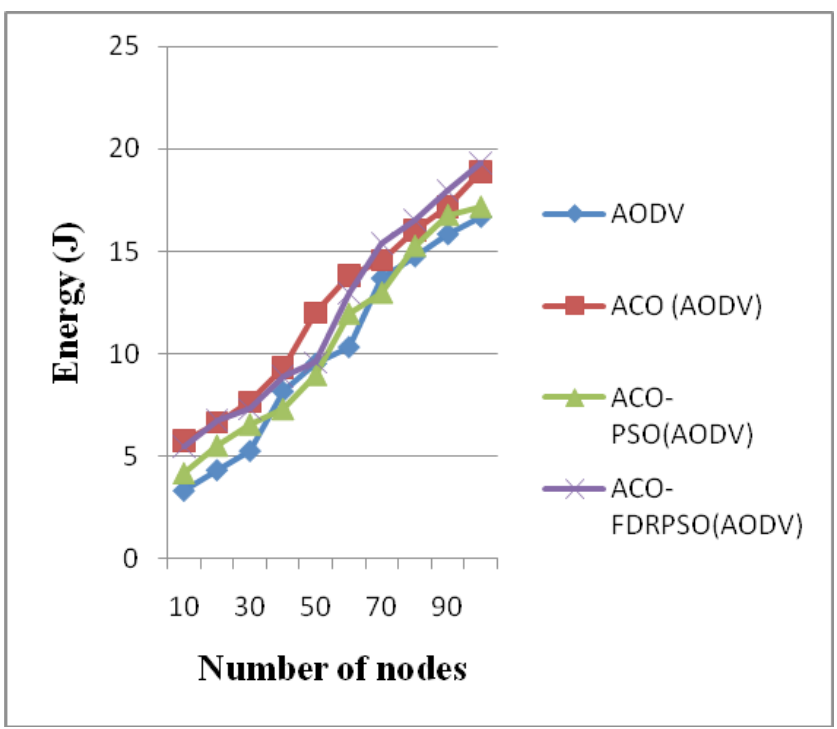

Figure 5. Energy comparison of conventional routing protocol with AI techniques.

\section{Conclusion}

This work proposed a hybrid ACO-FDR PSO technique along with conventional routing protocol for selecting optimal path with enhanced throughput. Hybrid ACOFDR PSO minimizes premature convergence issue and minimizes complexity at the time of pheromone update. The local solution is updated to a global best through limited iterations; the optimization function concentrates on retaining the transmission rates of the network. Besides, the hybrid approach minimizes the chances of retransmissions and transmission pause time that minimizes the network delay. The hybrid approach is simulated using NS2 software and the results are compared with AODV, ACO- PSO, and ACO-FDR PSO using throughput, delay, overhead, energy and PDR as parameters. The results evident that the throughput and PDR is increased by 2.52 and 1.01 times respectively than AODV protocol by the proposed hybrid method and delay is decreased by $62.28 \%$ than AODV protocol by the proposed hybrid method.

\section{References}

1. Royer EM, Toh CK. A review of current routing protocols for ad hoc mobile wireless networks, IEEE Personal Communications . 1999; 6(2):46-55. Crossref.

2. Sarkar SK, Basavaraju TG, Puttamadappa C. Ad hoc mobile wireless networks: Principles, protocols and applications. Auerbach Publications; 2007. Crossref.

3. Sharma M, Kansal M, Bhatia T. Simulation analysis of MANET routing protocols under different mobility models, International Journal of Wireless Communications and Networking Technologies. 2015; 4(1):1-8.

4. Karia DC, Godbole VV. New approach for routing in mobile ad-hoc networks based on ant colony optimisation with global positioning system, IET Networks. 2013; 2(1):171-80.

5. Tengviel J, Diawuo K, Dotche KA. The effect of the number of mobile nodes on varying speeds of manets, International Journal of Network Security and its Applications. 2012; 4(6):99-109.

6. Surendran S, Prakash S. An ACO look-ahead approach to QOS enabled fault-tolerant routing in MANETs, China Communications. 2015; 12(8):93-110. Crossref.

7. Younis O, Ghetie A, Eswaran S, Goldberg N, Samtani S, Varshney M, Patel M. A framework for evaluating network optimization techniques. Proceeding of 35th IEEE Sarnoff Symposium, Newyork: New Jersey; 2012. p. 1-5. Crossref.

8. Abbas NI, Ilkan M, Ozen, E. Fuzzy approach to improving route stability of the AODV routing protocol. EURASIP, Journal on Wireless Communications and Networking. 2015; 15(235):1-11. Crossref.

9. Rajan C, Shanthi N. Genetic based optimization for multicast routing algorithm for MANET, Indian Academy of Science. 2015; 40(8):2341-52.

10. Singh R, Singh DK, Kumar L. Swarm intelligence based approach for routing in mobile ad hoc networks, International Journal of Science and Technology Education Research. 2010; 1(7):147-53.

11. Jagdale BN, Patil P, Lahane P, Javale D. Analysis and comparison of distance vector, DSDV and AODV protocol of MANET, International Journal of Distributed and Parallel Systems. 2012; 3(2):121-31. Crossref.

12. Jha DK, Jain A, Jain S. AODV Performance optimization based multi-path routing in MANET, International Journal of Advances in Engineering and Technology. 2014; 7(2):508-12. 
13. Krishna PV, Saritha V, Vedha G, Bhiwal A, Chawla AS. Quality-of-service enabled ant colony-based multipath routing for mobile ad hoc networks, IET Communications. 2012; 6(1):76-83. Crossref.

14. Nancharaiah B, Mohan BC. The performance of a hybrid routing intelligent algorithm in a mobile ad hoc network, Computers and Electrical Engineering. 2014; 40(2):1255-64. Crossref.

15. Peram T, Veeramachaneni K, Mohan CK. Fitness-distanceratio based particle swarm optimization. Proceedings of the IEEE Swarm Intelligence Symposium; 2003. p. 174-81. Crossref.
16. Arif M, Rani T. ACO based routing for MANETS, International Journal of Wireless and Mobile Networks. 2012; 4(2):163-75. Crossref.

17. Caro GD, Ducatelle F, Gambardella LM. Ant colony optimization for routing in mobile ad hoc networks in urban environments. IDSIA Technical Report, Lugano: Switzerland; 2008.

18. Anitha M, Subramanian S, Gnanadass R. A Novel PSO algorithm for optimal production cost of the power producers with transient stability constraints, Journal of Electromagnetic Analysis and Applications. 2009; 1(4):265-74. Crossref.

19. Network simulator NS-2. Date accessed: 19/08/2012. http:// www.isi.edu/nsnam/ns/. 